Gas chromatography

late metabolic acidosis low birth weight mass spectrometry nutrition

premature infants transient tyrosinemia urea

\title{
Metabolic Studies of Transient Tyrosinemia in Premature Infants
}

\author{
S. A. FERNBACH, R. E. SUMMONS, W. E. PEREIRA, AND A. M. DUFFIELD
}

Departments of Pediatrics and Genetics. Stanford Medical Center, Stanford, California, USA

\section{Extract}

The recently developed technique of gas chromatographymass spectrometry supported by computer has considerably improved the analysis of physiologic fluids. This study attempted to demonstrate the value of this system in the investigation of metabolite patterns in urine in two metabolic problems of prematurity, transient tyrosinemia and late metabolic acidosis. Serial 24 -hr urine specimens were analyzed in 9 infants. Transient tyrosinemia, characterized by $5-10$ fold increases over basal excretion of tyrosine, p-hydroxyphenyllactate, and p-hydroxyphenylpyruvate in urine, was noted in five of the infants. Several infants had fluctuating levels of tyrosine metabolites in urine although dietary protein intake remained constant at $3-4 \mathrm{~g} / \mathrm{kg} / 24 \mathrm{hr}$ and ascorbic acid at $50 \mathrm{mg} / 24 \mathrm{hr}$. Late metabolic acidosis was seen in four infants, but bore no relation to transient tyrosinemia. The ratio of net acid to urea excretion in urine increased with increasing base deficit, implying a nonprotein origin of the metabolic acid. No unique metabolic patterns were characteristic of late metabolic acidosis.

\section{Speculation}

Advances in gas chromatography-mass spectrometry-computer technology (GC-MS-computer) allow for the screening of body fluids for a wide variety of compounds of biologic importance. One application of this technique is for the investigation of the nutritional adequacy of diets for premature infants. Clinical use of these tools may help establish parameters for assessment of nutrition and allow for a rationalization of diet.

There appear to be several defects and disturbances in the metabolism of tyrosine variously grouped under the names tyrosinemia, tyrosinosis, and tyrosyluria (17). One such metabolic defect commonly occurs in infants of low birth weight. Previous studies $(1,15,16,18-20)$ indicated that it is transient, aggravated by high protein (or high phenylalanine and tyrosine) intake, and that it is prevented by reducing protein intake or increasing vitamin $\mathrm{C}$ in the diet. This particular disturbance has been attributed to a functional insufficiency of the enzyme $p$-hydroxyphenylpyruvic acid oxidase, primarily due to incomplete development of the enzyme system complicated by substrate inhibition (1). In contrast, the enzymatic deficiency in hereditary tyrosinemia is unknown (17); however, taking into account the varied clinical patterns and metabolic profiles observed $(3,8,17,29)$ there are probably several different conditions being diagnosed under the name "tyrosinemia."

Serious clinical symptoms, such as failure to thrive, mental retardation, cirrhosis, renal tubular defects, and even death are associated with hereditary or permanent defects of tyrosine metabolism. Several follow-up studies of infants known to have suffered from transient tyrosinemia could not discern any harmful effects on neurologic or physical development in the first 2 years of life $(21,23)$. Recently, a long term follow-up study showed that the ultimate intellectual performance, particularly perceptual parameters, may be impaired in some low birth weight infants who had transient tyrosinemia in the neonatal period (22).

Late metabolic acidosis (LMA) of the premature, a disturbance of acid-base balance, has been well described in the literature $(2,4,9,13,14,24,26-28)$. We were impressed with several parallels between LMA and transient tyrosinemia. Both occur in prematures at 2-4 weeks of age. Severity and incidence are affected by protein intake and respond favorably to dietary management. Both are insidious without major symptoms and yet may adversely affect ultimate intellectual outcome.

The recently developed technique of GC-MS-computer has made considerable improvements to the tractability and specificity of the chemical analysis of physiologic fluids. It is now possible to identify and simultaneously quantitate many metabolites and to construct reliable profiles for various groups of compounds so that they might be used to characterize particular disease states $(2,4,9)$. The technique has recently been applied to the investigation of phenolic acids excreted in urine in hereditary tyrosinemia $(3,8)$ and to an investigation of phenylalanine-tyrosine metabolism where deuterium-substituted phenylalanine was used as a nonradioactive tracer (14).

The aims of this study were to demonstrate the value of combined GC-MS-computer techniques in the investigation of the relation between urinary metabolite patterns seen in two common metabolic defects of prematurity, late metabolic acidosis and transient tyrosinemia.

\section{MATERIALS AND METHODS}

The present study is based on a total of 104 24-hr urine samples from 16 premature or small for gestational age neonates, receiving treatment in the Stanford University Hospital Nursery. Criteria for entrance to the study included: birth weight less than $1,500 \mathrm{~g}$; hospitalization at Stanford within 1 day of birth; no complicating illnesses including respiratory distress syndrome, pneumonia, or documented sepsis. After exclusion of infants who became ill, died, or left the nursery, we were able to follow nine infants for periods of between 4 and 6 weeks from day 3 of life. All nine infants had birth weights of below $1,500 \mathrm{~g}$ and three were below $1,000 \mathrm{~g}$.

Infants were cared for according to routines at the Stanford Nursery. Incubators were used until the infants were able to maintain body temperature when they were moved to open cribs. All infants were fed Similac in either 20 or $24 \mathrm{cal} /$ ounce dilution. Diet was adjusted biweekly to maintain a constant protein intake of between 2.5 and $3.5 \mathrm{~g} / \mathrm{kg}$ body $\mathrm{wt} / 24 \mathrm{hr}$. 
The infants also received Tri-vi-sol, $0.6 \mathrm{ml} / 24 \mathrm{hr}$ (36 $\mathrm{mg}$ vitamin C) and iron (Fer-in-sol, $1.0 \mathrm{ml} / 24 \mathrm{hr}$ ).

All intake was recorded, as were daily weights. Urines were collected on ice three times/week using a plastic collection bag, and frozen after collection. Urea and creatinine in urine were determined by AutoAnalyzer. Twenty-four hour creatinine level in urine was used to assess completeness of urine collection. Titratable acidity, urinary $\mathrm{NH}_{4}$, and net acid excretion were performed by the method of Kildeberg (14).

Blood gas determinations were from arterialized capillary samples and performed three times per week at the conclusion of each 24-hr urine collection period, with the use of a Radiometer or Corning analyzer. Base excess was calculated using the Severinghaus calculator, correcting for hemoglobin levels. Using the criteria of Svenningsen and Linquist (28), an infant was clsssified as having LMA if after the first week of life, base deficit was $8 \mathrm{mEq}$ or greater for two determinations in succession.

Gas liquid chromatography was carried out using a Varian 2100 gas chromatograph equipped with dual $6 \mathrm{ft}$ by $1 / 8$ inch (inner diameter) U-shaped glass columns packed with $10 \%$ OV-17 on Gas Chrom Q (no. 100-120). Nitrogen was used as carrier gas (flow, $25 \mathrm{ml} / \mathrm{min}$ ) and the oven was programmed at $4 \%$ min after a 6 -min delay at the injection temperature of $100^{\circ}$. The gas chromatograph was equipped with dual flame ionization detectors and the signal was recorded using a Brush Mark 280 dual pen recorder.

Gas chromatography-mass spectrometry was carried out using a Varian 1200 gas chromatograph coupled via an all glass line and a silicone membrane separator to a Finnigan 1015 quadrupole mass spectrometer which in turn was interfaced to the ACME computer system at Stanford University Medical School (28). Gas-liquid chromatography separations on this system were carried out using a coiled glass column ( $6 \mathrm{ft}$ by $1 / 8$ inch) packed with $10 \%$ OV-17 on Gas Chrom Q (no. $80-100$ ), using helium as carrier gas and operated under the same conditions as above.

$\mathrm{HCl}(1.25 \mathrm{~N})$ in 1-butanol and $25 \%(\mathrm{v} / \mathrm{v})$ trifluoroacetic anhydride in methylene chloride were obtained from Regis Chemical Co., Ill. Etheral diazomethane was prepared from Diazald $\mathrm{R}$ from Aldrich Chemical Co. using the method given by Fieser and Fieser (7). 2-Amino-octanoic acid was obtained from Eastman Organic Chemicals, N.Y., and n-tetracosane and column packings from Applied Science Laboratories, $\mathrm{Pa}$.

Urine $(2.5 \mathrm{ml})$ was acidified to $\mathrm{pH} 1$ using $1 \mathrm{~N} \mathrm{HCl} ; 50 \mu 1$ tetracosane $(1 \mathrm{mg} / \mathrm{ml})$ and $50 \mu 1 \mathrm{DL}$-2-aminooctanoic acid (1 $\mathrm{mg} / \mathrm{ml}$ ) were added as internal standards. The urine was then extracted with $3 \times 5 \mathrm{ml}$ ethyl acetate and $1 \times 5 \mathrm{ml}$ ether. The combined organic phase was dried with anhydrous sodium sulfate and evaported in vacuo. The residue, in methanol-ether solution, was methylated with excess ethereal diazomethane overnight. The solution was again evaported in vacuo and the residue taken up in $200 \mu 1$ injected on one column of the gas chromatograph.

The aqueous solution remaining after extraction $(0.5 \mathrm{ml})$ was placed on a $5-\mathrm{ml}$ bed volume column of Bio-Rad AG-50W-X12 cation exchange resin. After washing with $20 \mathrm{ml}$ water the amino acids were eluted with $20 \mathrm{ml} 3 \mathrm{~N}$ ammonia solution. The eluate was evaporated to dryness in vacuo, refluxed with $2 \mathrm{ml} 1.25 \mathrm{~N} \mathrm{HCl}$ in 1-butanol for $15 \mathrm{~min}$ and again evaporated to dryness in vacuo. Trifluoroacetic anhydride in methylene chloride $(25 \% \mathrm{v} / \mathrm{v}, 0.5 \mathrm{ml})$ was added to the residue and the solution heated at $100^{\circ}$ in a sealed vial for 15 min. An aliquot $(1-2 \mu 1)$ of this solution was injected on the second column of the gas chromatograph simultaneously with the methylated acids. Quantitation of the various metabolites was made by comparing the peak heights with those of the internal standards and with adjustments made for the respective sensitivity factors determined by calibration with known amounts of each compound. The identities of the various compounds in the gas-chromatographic profile were initially determined and subsequently checked by combined GC-MS although relative retention times proved to be fairly reliable for metabolite identification.

\section{RESULTS}

Transient tyrosinemia, defined by the presence of at least 10 $\mathrm{mg} / \mathrm{kg} / 24 \mathrm{hr}$ of tyrosine and its metabolites, was detected in five of the nine infants studied. It was characterized by a marked elevation in the excretion in urine of the tyrosine metabolites $p$-hydroxyphenyllactic acid, $p$-hydroxyphenylpyruvic acid, and $p$-hydroxyphenylacetic acid to levels of at least $5,0.5$, and $0.5 \mathrm{mg} / \mathrm{kg}$, respectively. There was also a less marked but distinct elevation in the tyrosine output in urine. Figures 1 and 2 show the metabolic profiles of the same infant $(J L)$ in the normal $(a)$ and tyrosinemic $(b)$ states. Figure 1 shows the free acid outputs, chromatographed as the methyl ester-methyl ether derivatives and Figure 2 is a profile of the free amino acids of the same urines, chromatographed as the

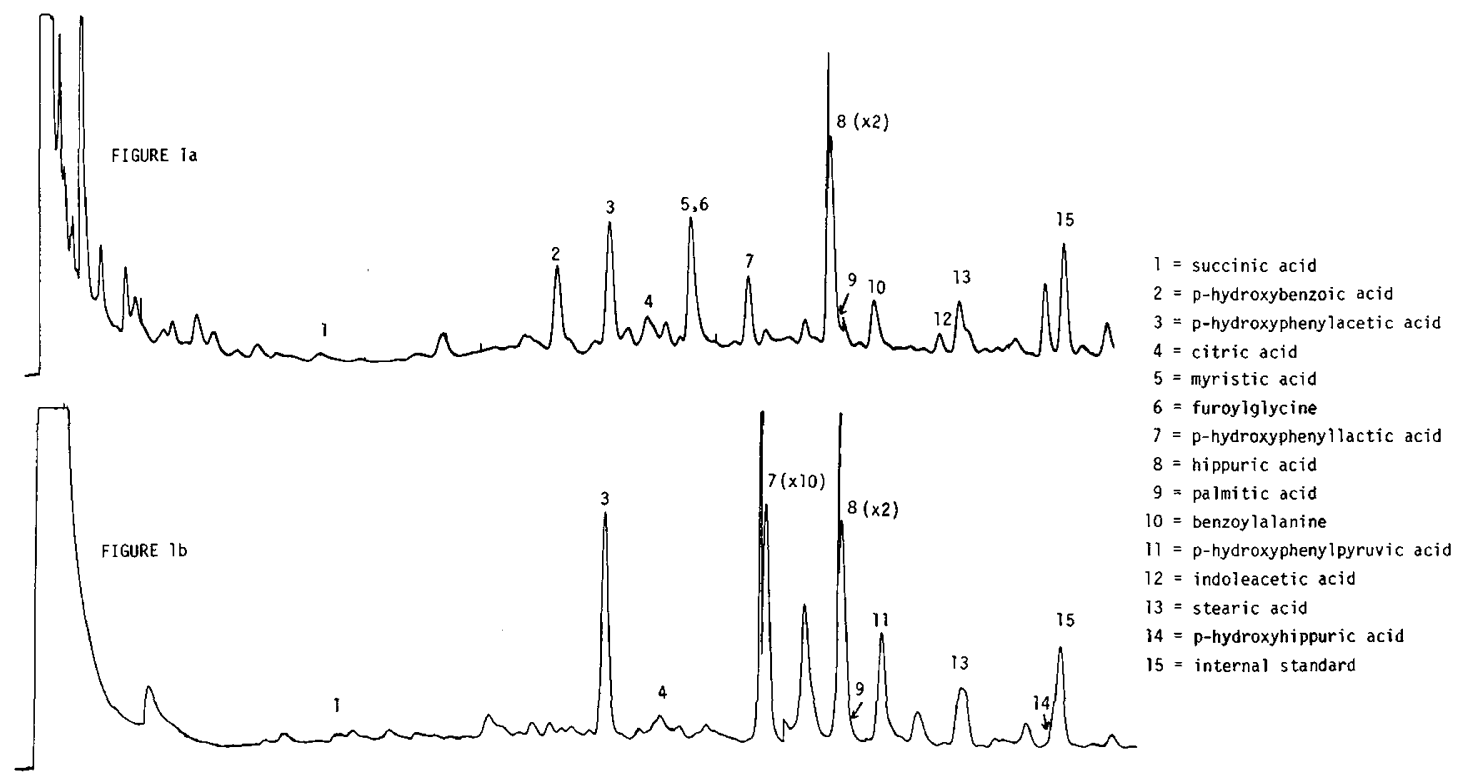

Fig. 1. Comparison of chromatographic profiles of urinary free acids for an infant $(J L)$ in the normal $(a)$ and tyrosinemic $(b)$ states. $p$-Hydroxyphenyllactic acid is markedly elevated in the latter. 


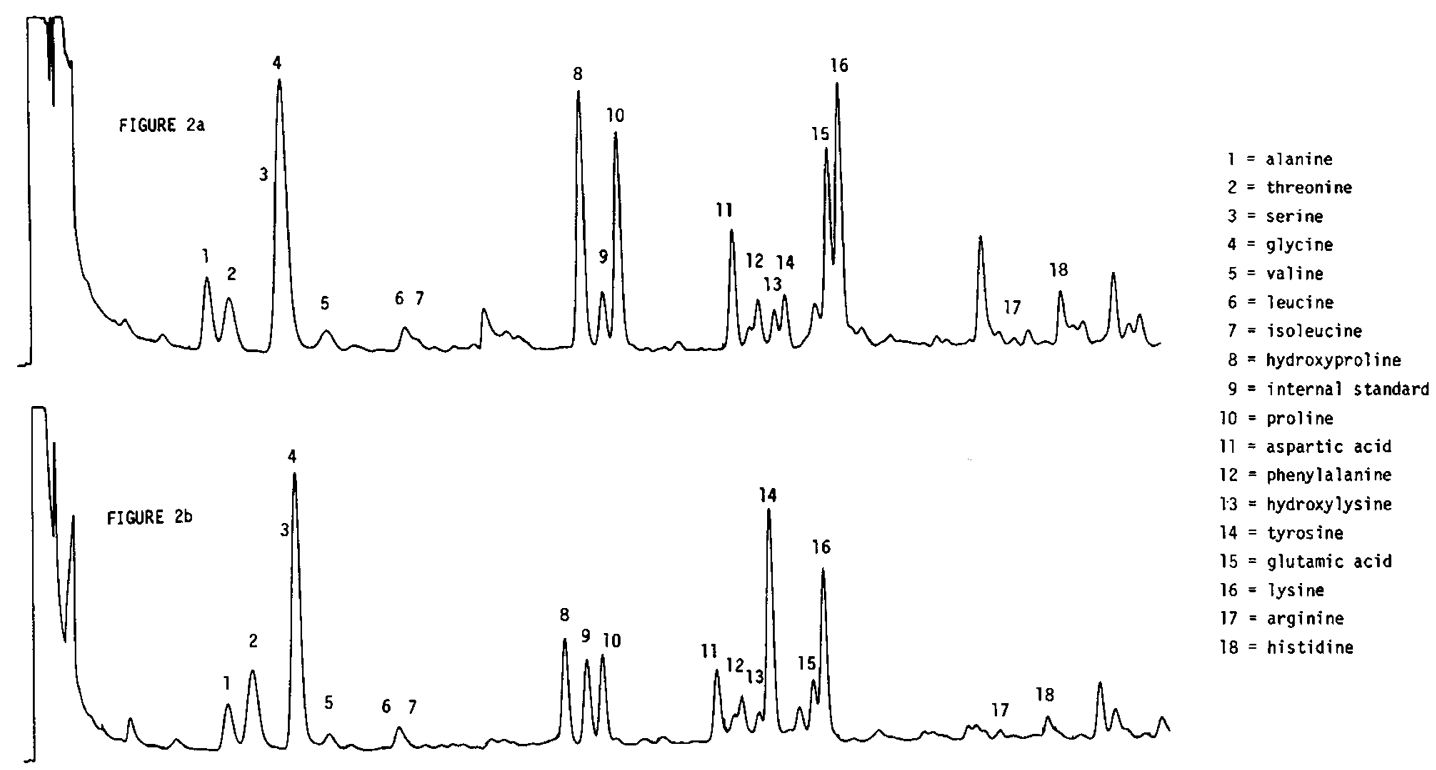

Fig. 2. Comparison of chromatographic profiles of urinary free amino acids for an infant $(J L)$ in the normal $(a)$ and tyrosinemic $(b)$ states. There is a threefold elevation of tyrosine in the latter.

$N$-trifluoroacetyl $n$-butyl ester derivatives. In each case the concentration of each metabolite is a function of the peak height as compared with the height of the internal standard. Table 1 is a summary of the ranges of output of tyrosine and metabolites in urine observed for all the infants in the study.

As shown by Table 1 and Figure 1 neonatal tyrosinemia is characterized by a very large increase in the output of $p$-hydroxyphenyllactic acid and by a 10-50-fold excess of the latter over $p$-hydroxyphenylpyruvic acid. Studies of the hereditary defects in tyrosine metabolism initially indicated that $p$-hydroxyphenylpyruvic acid was the major metabolite (17, 29), although more recently Wadman et al. (29), Bodegard et al. (3), and Crawhall et al. (5) reported cases in which $p$-hydroxyphenyllactic is in a $2-5$-fold excess over $p$-hydroxy phenylpyruvic. The latter determinations were made using GC and GC-MS methods, and probably reflect the improved specificity of the analytical procedure over the previously used colorimetric methods rather than a difference in the actual metabolic profile. Apart from the very large excess of $p$-hydroxyphenyllactic acid over its keto analog we could detect no significant differences between the profiles shown in neonatal tyrosinemia and those published for hereditary tyrosinemia $(3,5,29)$. Bodegard et al. (3) also compared urinary acid profiles of hereditary tyrosinemia and the transient defect and were also unable to detect any gross features which would allow easy differentiation of the two syndromes. Other metabolites such as p-hydroxymandelic acid, Dopa, $N$-acetyltyrosine, which have previously been reported in tyrosinemic urine $(1,17)$, were not elevated.

Figure 3 compares the excretion of phenolic acids over an extended period of four infants who showed transient tyrosinemia. In following the transient defect over an extended pariod, we have observed rapid fluctuations in the tyrosine metabolite output measured in complete urine collections despite uniform diet and constant environmental

Table 1. Daily excretion of tyrosine and its metabolities $(\mathrm{mg} / \mathrm{kg})^{1}$

Tyrosine $\quad p$-hp-lactate $\quad p$-hp-pyruvate $p$-hp-acetate

\begin{tabular}{lcccc}
\hline Normal & $0.2-3$ & $0-5$ & $0-0.5$ & $0.2-2$ \\
Tyrosinemic & $3-15$ & $5-50$ & $0.5-5$ & $0.5-5$ \\
\hline
\end{tabular}

${ }^{1}$ hp: hydroxyphenyl-.

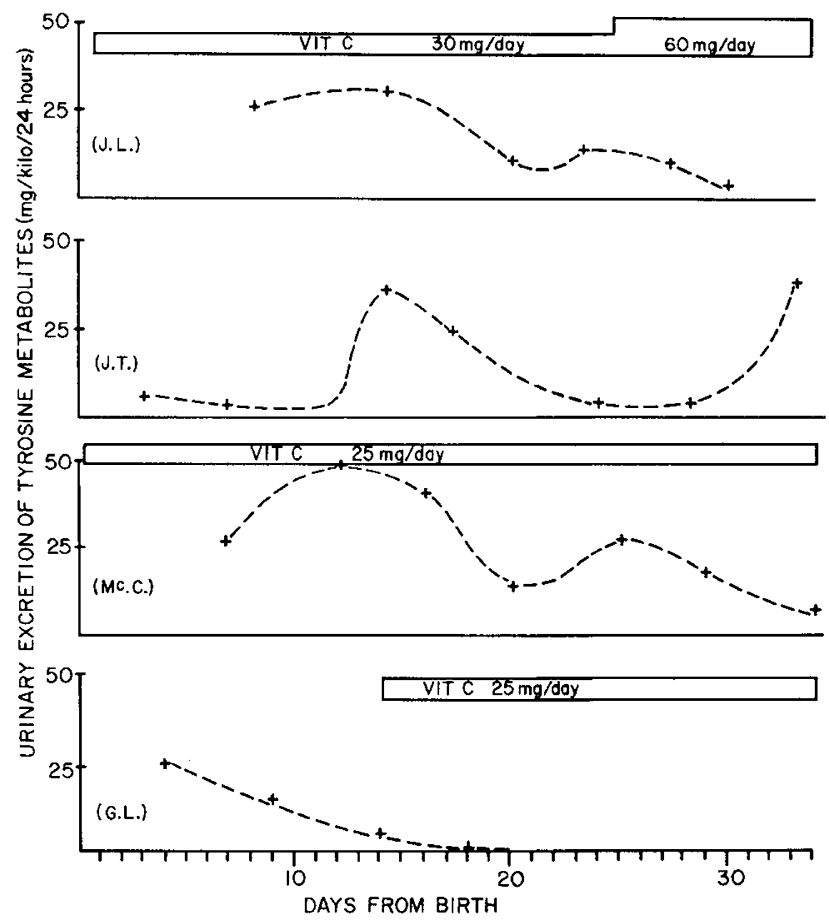

Fig. 3. Comparison of combined daily excretion of the tyrosine metabolites $p$-hydroxyphenyllactic acid and $p$-hydroxyphenylpyruvic acid for four infants who developed transient tyrosinemia while receiving usual doses of vitamin $\mathrm{C}($ VIT C).

influences in the nursery. We feel that this disturbance is recognized reliably only by repeated observation for these compounds. In contrast, the hereditary defect would be expected to show persistent excretion of phenolic acids.

The addition of $50 \mathrm{mg} / \mathrm{kg}$ supplementary vitamin $\mathrm{C}$ was associated with the termination of tyrosinemia in the four patients given this higher dose. The spontaneous fluctuation in phenolic excretory products does not allow us to comment on the adequacy of this amount of vitamin $\mathrm{C}$ although none of the patients treated showed a recurrence.

Late metabolic acidosis was observed in four of the patients. Of these, three were also found to have transient tyrosinemia. 

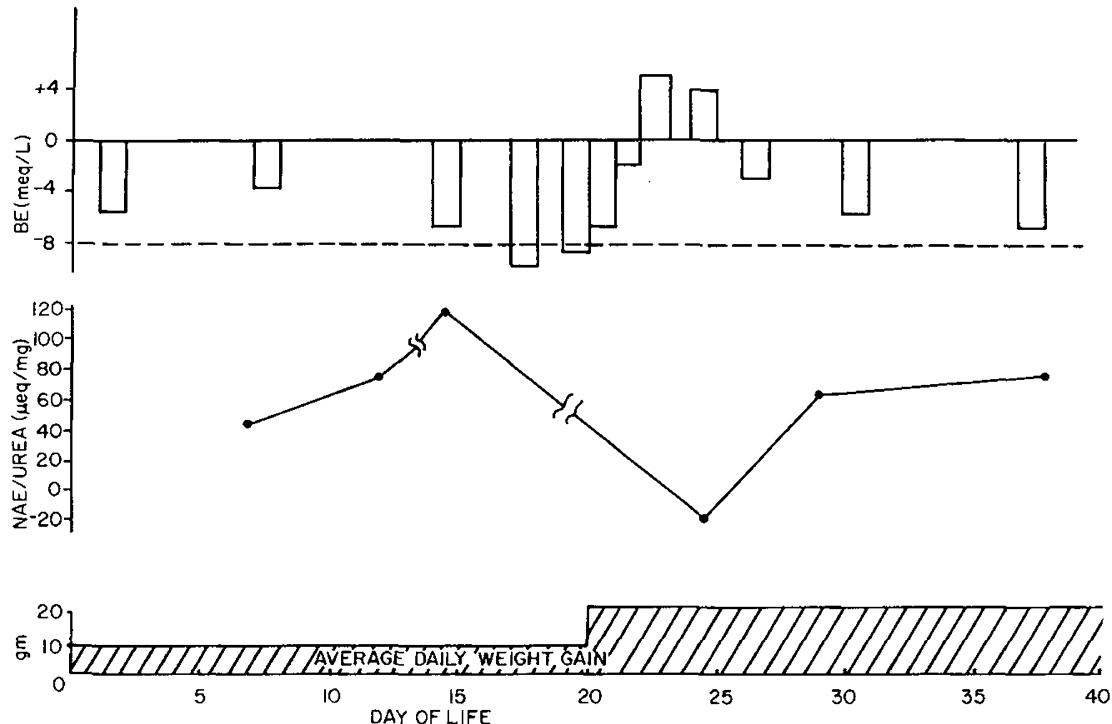

Fig. 4. Base excess, net acid excretion (NAE) to urea ratio (microequivalents per milligram) and average daily weight gain for patient $M c C$.

In those infants without LMA, two of five were noted to have tyrosinemia.

Figure 4 represents typical findings in our patients with late metabolic acidosis (this is patient $M c C$ of Fig. 3). Protein intake averaged $3 \mathrm{~g} / \mathrm{kg} / 24 \mathrm{hr}$. Weight gain was constant throughout the period but increased in rate after the termination of the episode of acidosis. Urea excretion remained constant at $125-150 \mathrm{mg} / \mathrm{kg} / 24 \mathrm{hr}$. Net acid excretion (NAE) increased during the period of developing acidosis, remained at a high level during the acidotic episode, then returned to a lower level upon resolution. The increasing NAE to urea ratios during the development of the acidosis imply either altered pathways of protein catabolism, or nonprotein origin for the acid.

Apart from a surprising incidence of transient tyrosinemia in those patients with LMA, we were unable to detect any consistent or unique excretory patterns which might suggest an etiology. In addition, there was no correlation between the time course or onset of LMA and transient tyrosinemia.

\section{DISCUSSION}

The improvement in survival of small premature infants as a result of advances in neonatal intensive care has placed new demands on the pediatrician. To ensure survival of an intact infant, the physician must essentially assume the placental functions of oxygenation and nutrition. The control of respiratory function depends on the constant monitoring of blood gases. Unfortunately, no such data is available for the assessment of the adequacy or propriety of nutrition. As a result, small premature infants are fed diets known to be reasonably appropriate for full term infants; if weight gain is maximal, the diet is considered good. There is ample evidence that this conclusion may be fallacious $(1,2,4,9,13,14,22$, $24,26-28$ ).

The development of GC-MS-computer systems allows for the screening of a blood or urine sample for a wide variety of organic compounds. We feel that the use of such systems will permit the accumulation of data essential for the optimal dietary management of the premature.

We have applied this technique to premature neonates fed a standard diet with standard vitamin supplementation. We were interested to find wide fluctuations in phenolic excretory products in spite of constant intake. When this variation was considered, over $50 \%$ of infants in this small series demonstrated transient tyrosinemia. This is a significantly higher incidence than previously reported (1), and implies that standard diets for prematures are seriously deficient in vitamin C.

This finding assumes more than academic interest when the morbidity of this diet-accentuated enzymatic inadequacy is considered. Although Avery (1) reports few detectable symptoms in the newborn period, and others $(21,23)$, no symptoms in the first 2 years, a follow-up study by Menkes and coworkers (22) demonstrated significant perceptual handicaps in the patients followed. We are dealing, then, with a nutritional disease of the newborn which may be "silent" until higher intellectual functions can be tested.

We have also used this method to investigate a possible correlation between late metabolic acidosis and transient tyrosinemia. Our data clearly indicated there to be no such association. In addition, no unique metabolites were associated with the development of LMA.

We would advocate continuing research into the application of GC-MS-computer systems to investigate and monitor the nutritional status of the neonate. We feel that the establishment of normal and abnormal excretory patterns will enable the physician to rationalize and individualize dietary management of the small infant.

\section{SUMMARY}

Serial 24-hr urine specimens were analyzed in nine infants by gas chromatography-mass spectrometry. Transient tyrosinemia was observed in five of the infants. This syndrome was characterized by a 5-10-fold increase over basal excretion of tyrosine, $p$-hydroxyphenyllactic, and $p$-hydroxyphenylpyruvic acids in urine. Late metabolic acidosis was seen in four of the infants studied. Late metabolic acidosis bore no relation to transient tyrosinemia and no metabolic signature characteristic of LMA was observed.

\section{REFERENCES AND NOTES}

1. Avery, M. E., Clow, C. L., Menkes, J. H., Ramos, A., Scriver, C. R., Stern, L., and Wasserman, B. P.: Transient tyrosinemia of the newborn: Dietary and clinical aspects. Pediatrics, 39: 378 (1967).

2. Ballabriga, A.: Study of the acid-base balance of the premature infant during various diets. Helv. Paediat. Acta, 20: 527 (1965).

3. Bodegard, G., Gentz, J., Lindblad, B., Lindstedt, S., and Zetterstrom, R.: Hereditary tyrosinemia. III. On the differential diagnosis and the lack of effect of early dietary treatment. Acta Pediat. Scand., 58: 37 (1969).

4. Bucci, G., Scalamandre, A., Savignoni, P. G., and Mendicini, M. Acid-base status of normal premature infants in the first weeks of life. Biol. Neonatorum, 8: 81 (1965). 
5. Crawhall, J. C., Mamer, O., Tjoa, S., and Claveau, J. C.: Urinary phenolic acids in tyrosinemia. Identification and quantitation by gas chromatography-mass spectrometry. Clin. Chim. Acta, 34: 47 (1971).

6. Curtius, H.-Ch, Vollmin, J. A., and Baerlocher, K.: The use of deuterated phenylalanine for the elucidation of the phenylalanine-ty rosine metabolism. Clin. Chim. Acta, 37: 277 (1972).

7. Fieser, L. F., and Fieser, M.: Reagents for Organic Synthesis, p. 191 (John Wiley \& Sons, Inc., 1967).

8. Gentz, J., Lindblad, B., Lindsteds, S., and Zetterstrom, R.: Studies on the metabolism of the gas-liquid chromatographic method. J. Lab. Clin. Med., 74: 185 (1969).

9. Glick, H., and Allen, A. C.: Diet-induced metabolic acidosis in prematurely born infants, J. Pediat., 78: 1061 (1971).

10. Horning, E. C., and Horning, M. G.: Human metabolic profiles obtained by GC and GC/MS. J. Chromatogr. Sci., 9: 129 (1971).

11. Horning, E. C., and Horning, M. G.: Metabolic profiles: gas-phase methods for analysis of metabolites. Clin. Chem., 17: 802 (1971).

12. Jellum, E., Stokke, O., and Eldjarn, L.: Screening for metabolic disorders using gas-liquid chromatography mass spectrometry and computer technique. Scand. J. Clin. Lab. Invest., 27: 273 (1971).

13. Kildeberg, P.: Clinical acid base physiology. In: Studies in Neonates, Infants and Young Children (Munksgaard, Copenhagen, 1968).

14. Kildeberg, P.: Disturbances of hydrogenion homeostasis. II. Late metabolic acidosis. Acta Paediat. Scand., 53: 517 (1964).

15. Kretchmer, N.: Enzymatic patterns during development. Pediatrics, 23: 606 (1959).

16. Kretchmer, N., Levine, S. Z., and McNamara, H.: Certain aspects of tyrosine metabolism in the young. (I). The development of the tyrosine oxidising system in human liver. J. Clin. Invest., 2: 236 (1965).

17. La Du, B. N., and Gjessing, L. R.: Tyrosinosis and Tyrosinemia in The Metabolic Basis of Inherited Disease, Ed. 3, Chap. 12 (McGraw-Hill Book Co., 1972).

18. Levine, S. Z., Dann, M., and Marples, E.: A defect in tyrosine and phenylalanine metabolism in premature infants. (III). Demonstration of the irreversible conversion of phenylalanine to tyrosine in the human organism. J. Clin. Invest., 22: 551 (1943).

19. Levine, S. Z., Gordon, H. H., and Marples, E.: A defect in tyrosine and phenylalanine metabolism in premature infants. (ii). Spontaneous occurrence and eradication by vitamin C. J. Clin. Invest., 20: 209 (1941).

Copyright $(1975$ International Pediatric Research Foundation, Inc.
20. Levine, S. Z., Marples, E., and Gordon, H. H.: A defect in tyrosine and phenylalanine metabolism in premature infants. (I). Identification and assay of intermediary products. J. Clin. Invest., 20: 199 (1941).

21. Menkes, J. H., Chernick, V., and Ringel, B.: Effect of elevated tyrosine on subsequent intellectual development of premature infants. J. Pediat., 69: 583 (1966).

22. Menkes, J. H., Welcher, D. W., Levi, H. S., Dallas, J., and Gretsky, N. E.: Relationship of elevated blood tyrosine to the ultimate intellectual performance of premature infants. Pediatrics, 49: 218 (1972).

23. Partlington, M. W., Delahaye, D. J., Masotti, R. E., Read, J. H., and Roberts, B.: Neonatal tyrosinemia: A follow-up study. Arch. Dis. Childhood, 43: 195 (1968).

24. Ranlov, P., and Siggaard-Anderson, O.: Late metabolic acidosis in premature infants. Acta Paediat. Scand., 54: 531 (1965).

25. Reynolds, W. E., Bacon, V. A., Bridges, J. C., Coburn, T. C., Halpern, B., Lederberg, J., Levinthal, E. C., Steed, E., and Tucher, R. B.: A computer operated mass spectrometer system. Anal. Chem., 42: 1122 (1970).

26. Schain, R. J., and O'Brien, K.: Longitudinal studies of acid-base status in infants with low birth weight. J. Pediat., 70: 885 (1967).

27. Svenningsen, N. W.: Metabolic acidosis in preterm infants during the first weeks of life. Acta Paediat. Scand., 56: 439 (1967).

28. Svenningsen, N. W., and Lindquist, B.: Incidence of metabolic acidosis in term, preterm and small-for-gestational age infants in relation to dietary protein intake. Acta Paediat. Scand., 62: 1 (1973).

29. Wadman, S. K., Van Sprang, F. J., Mass, J. W., and Ketting, D.: An exceptional case of tyrosinosis. J. Ment. Defic. Res., 12: 269 (1968).

30. This work was supported by a grant from the Manned Space Center, NASA (Grant no. NGR 05-020-632) and RR-00612, from the National Institutes of Health, Grant no. RR-81 from the General Clinical Research Centers Program of the Divisions of Research Resources, National Institutes of Health, Research Grant no. HD-02147, and Training Grant no. HD-00049 from the National Institutes of Health.

31. Requests for reprints should be addressed to: A. Duffield, Ph.D., Department of Genetics, Stanford University Medical Center, 300 Pasteur Dr., Stanford, Calif. 94305 (USA).

32. Accepted for publication December 3, 1974.

\title{
Sex Differential of Survival during Perinatal Period: An Immunologic Phenomenon?
}

\author{
MICHEL C. VERNIER(36) \\ Division of Epidemiology, School of Public Health, University of California, Berkeley, California, USA
}

Extract

The sex-differential early neonatal mortality experienced by a population of 27,243 neonates has been analyzed in relation to sex-related reproductive antecedents of their mothers. An association between the risk of dying of a neonate and the sex of previous pregnancies of its mother has been found: the risk decreases with a previous pregnancy of the same sex as the neonate, and increases with one of the opposite sex. Repeated pregnancies of same sex increase the phenomenon. An immunologic hypothesis has been formulated from these results: sex-differential survival of conceptuses during the perinatal period would be due to sex-differential conceptomaternal immunologic interactions. An advantage in survival of the female fetus results from these interactions in primiparous mothers. However, immunologic behavior of the mother is 